van der Laan: Would Dr Schmidt care to comment on the results of Green and himself in the light of $\mathrm{Dr}$ Bolton's results?

Schmidt: The steep gradient of optical counts of quasars with magnitude, between the Green-Schmidt $(B<15.7)$ and the Braccesi $\left(\mathrm{B}<1 \mathrm{~m}^{\mathrm{m}} \mathrm{0}\right)$ surveys, applies only to objects with ultraviolet excess, since quasars in both surveys fulfil (U-B) $<-0.5$. We can say nothing as yet about the quasars with redder (U-B) colours (with redshifts less than 2.5), if they exist.

Bolton: In our 2-hour field non UV excess objects with strong Mg II in the $\mathrm{B}$ band make an appreciable contribution to the low redshift numbers.

Tammann: I would like to add that Dr Stepe at Basle has been using UG as well as UV plates to search for ultraviolet excess objects. Using the UG plates which are taken with narrower filters than the UV plates, he finds $20 \%$ more ultraviolet excess objects than with the normal UV plates, in agreement with what you said.

Khachikian: I should like to point out that there are also strong differences between Markarian galaxies which have strong UV continuum and those which have not. The galaxies with strong UV continuum usually have active nuclei and most of them are the Seyfert galaxies.

\title{
A SURVEY PROGRAMIE FOR QSO AND RELATED OBJECTS
}

\section{H. Lorenz}

I would like to give the first results of a survey programme for high redshift quasars and related objects on the Tautenburg-Schmidt plates. It is known that objective prism plates are very useful for detecting these objects. The smaller the dispersion of the spectra, the fainter are the objects that can be found. Our Schmidt correcting lens gives spectra with the dispersion of $2500 \AA \mathrm{mm}^{-1}$ at $\mathrm{H} \gamma$. The limiting magnitude is about $19^{\mathrm{m}}$ in the $\mathrm{B}$ range. At this very low dispersion reliable classification of the objects requires the extraction of all the information stored on the plate. That means that a quantitative evaluation should supplement visual insepction.

First we detrmine the wavelength of spectral features in the suspected object using a wavelength scale, which has been determined by the position of reference stars on a direct Schmidt plate and the position of spectral features of these stars on the prism plate. The accuracy is sufficient to determine redshifts larger than 0.04 . The main purpose is the determination of the intensity distribution in the spectra. The characteristic curve (as a function of $\lambda$ ) is derived from the known average intensity distributions in stars of the same spectral types as the references. The relatively crude estimate of the spectral 
types is compensated by the large number ( $\sim 30)$ of stars. The measurements of the plates are performed on an automatic microdensitometer.

Up to now we have surveyed visually a field of 90 square degrees and found 15 emission 1 ine objects. If these are really quasars the surface density is 0.16 per square degree. The first application of the method to these quasars described above proves that the quantitative analysis of very low dispersion spectra increases considerably the power of visual inspection. 